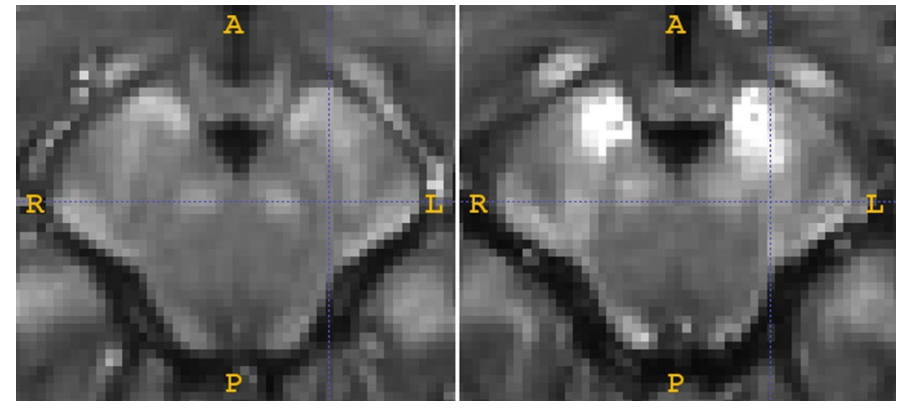

$\mathrm{T} 1 \mathrm{w} / \mathrm{T} 2 \mathrm{w}$ ratio map showing a higher ratio (white) in the substantia nigra pars compacta of a patient with PD (right) than in a healthy control (left). Image courtesy of G. Du and X. Huang, Pennsylvania State University, USA

with PD and 87 healthy controls. In addition, logistic regression analysis showed that the T1w/T2w ratio in the $\mathrm{SNc}$ was a significant predictor to distinguish between patients with PD and healthy controls.

"Our results suggest that the $\mathrm{T} 1 \mathrm{w} / \mathrm{T} 2 \mathrm{w}$ ratio sensitively discriminates early-stage PD patients from controls," says Du. "We now aim to establish normed scores for

the T1w/T2w ratio sensitively discriminates early-stage PD patients from controls

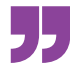

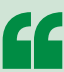

These findings

... could help

explain the

development

of cognitive

symptoms

in Down

syndrome implementation in multisite clinical and research platforms to aid in the early diagnosis of PD patients."

Ian Fyfe

ORIGINAL ARTICLE Du, G. et al. Magnetic resonance $\mathrm{T} 1 \mathrm{w} / \mathrm{T} 2 \mathrm{w}$ ratio: a parsimonious marker for Parkinon's disease. Ann. Neurol. https://doi.org/ 10.1002/ana.25376 (2018)

Compared with cells from healthy controls, neurons from individuals with Down syndrome showed increased dendritic spine stability and density, and also had a reduced capacity to communicate and form functional neural networks. "These findings are important, because they could help explain the development of cognitive symptoms in Down syndrome," comments Raquel Real, the co-first author of the study.

"The immediate next step is to understand these defects at the molecular level, and we will be looking into which genes and pathways are involved - we have already identified a few promising candidates," says De Paola. "In the long term, we plan to use this system to model other complex genetic conditions such as autism and Heather Wood

ORIGINAL ARTICLES Real, R. N. et al. In vivo modelling of human neuron dynamics and Down syndrome. Science 362, eaau1810 (2018) Hithersay, R. et al. Association of dementia with mortality among adults with Down syndrome older than 35 years. JAMA Neurol. https://doi.org/ 10.1001/jamaneurol.2018.3616 (2018) schizophrenia, as well as dementia."

\title{
Oligodendrocytes - active accomplices in MS pathogenesis?
}

Oligodendrocytes might acquire immune cell-like properties in multiple sclerosis (MS), according to new research in mice. The new findings suggest that these cells play a more active part in MS pathogenesis than previously thought.

In patients with MS, autoimmune attack on myelin leads to a loss of oligodendrocytes. The prevailing view is that MS pathogenesis begins in the peripheral immune system and that myelin becomes a passive victim of this autoimmune dysfunction.

"We were surprised to find that a subset of oligodendrocyte lineage cells, particularly oligodendrocyte precursor cells (OPCs), acquire immunological properties in MS," explains Ana Mendanha Falcão, lead author of the new report. "Our study suggests that oligodendrocytes and OPCs are not passive targets but instead active immunomodulators of the disease."

Falcão and colleagues examined oligodendrocytes and OPCs from mice with experimental autoimmune encephalomyelitis (EAE), a model of MS, and from healthy control mice. The team employed a new technology called single-cell RNA sequencing to characterize the transcriptomes of oligodendrocyte lineage cells extracted from the spinal cords of these mice. Intriguingly, cells from EAE mice included populations of oligodendrocytes with unique transcriptional states that were not found in healthy control mice.

Further characterization of these EAE-enriched cell populations revealed subsets of oligodendrocytes that express genes ordinarily associated with immune cells, including genes encoding components of major histocompatibility complex (MHC) I and II. MHC-II-expressing OPCs were able to phagocytose myelin and to activate memory and effector $T$ lymphocytes, suggesting that these cells play an active part in the autoimmune response in MS.

To examine the relevance of these findings in humans, the team examined post-mortem brains from patients who had MS. They confirmed that MHC-II was expressed in a population of human oligodendrocytes in the context of disease. Furthermore, expression of many genes that have previously been linked to MS susceptibility in humans was increased in oligodendrocyte lineage cells in the mouse EAE model.

These findings suggest that oligodendrocyte lineage cells have a greater role in the origin and progression of MS than previously thought. "We are now investigating what the functions of immunocompetent oligodendroglia are, and whether we can modulate their mode of action," concludes senior author Gonçalo Castelo-Branco. "Most current therapies for MS target the immune system, but approaches that modify the immunomodulatory properties of oligodendrocytes and their precursors might lead to improved approaches in combination with current therapies."

Charlotte Ridler

ORIGINAL ARTICLE Falcão, A. M. et al. Disease-specific oligodendrocyte lineage cells arise in multiple sclerosis. Nat. Med. https://doi.org/10.1038/s41591-018-0236-y (2018) 